\title{
Monitoring and Predicting Response to Peptide Receptor Radionuclide Therapy: A Quantitative SPECT-CT Based Analysis
}

\author{
Ponraj Chinnadurai ${ }^{1,2,}$, Scott Berg ${ }^{1, \S}$, Guillaume Nicolas ${ }^{1,}{ }^{*}$, Martin Walter $^{1}$, Helmut Rasch ${ }^{1}$, \\ Jan Mueller-Brand ${ }^{1}$ and Stefan Kneifel ${ }^{1}$ \\ ${ }^{I}$ Department of Nuclear Medicine, University Hospital, Basel, Switzerland \\ ${ }^{2}$ School of Medical Science and Technology, Indian Institute of Technology, Kharagpur, India
}

\begin{abstract}
Purpose: A standard imaging protocol for monitoring and predicting the response to Peptide Receptor Radionuclide Therapy (PRRT) has not been established yet. The aim of this study was to evaluate the response to PRRT by quantitative SPECT-CT and assess the value of SPECT-CT over routine planar scintigraphy in predicting the outcome of PRRT.

Methods: 32 patients with neuroendocrine tumour, who underwent SPECT-CT imaging after the first and second course of radiolabelled DOTATOC were retrospectively analysed. The response to therapy was defined by decrease in tumour volume in CT. The uptake in planar scintigraphy was evaluated by visual assessment based on a 5-point scoring scale. From SPECT images, ratios of maximum and mean values of tumour uptake to normal liver uptake were calculated. The value of SPECT-CT over planar scintigraphy in predicting therapy response was assessed by Receiver Operating Characteristic (ROC) analysis.

Results: Evaluation of 77 target lesions (in 32 patients) by SPECT-CT imaging, showed that the mean uptake ratio decreased from a median value of 5.66 (range 0.5 to 32.37 ) to 3.85 (range 0.95 to 15.75 ), with an overall $26 \%$ decrease (P $<0.001)$. The maximum uptake ratio decreased from a median value of 7.98 (0.97 to 54.21) to 5.59 (1.95 to 29.71), with an overall $30 \%$ decrease $(\mathrm{P}<0.002)$. On evaluation of 67 measurable lesions by CT (in 23 patients), the tumour volume decreased from a median value of $58.5 \mathrm{~cm}^{3}(14.1$ to 411.7$)$ to $38.3 \mathrm{~cm}^{3}(9.9$ to 428.4$)$ with an overall $35 \%$ decrease $(\mathrm{P}=$ 0.001). The area under the ROC curve was 0.733 (95\% Confidence Interval: $0.203,0.819)$ for SPECT-CT and 0.551 (95 $\%$ Confidence Interval: $0.485,0.981)$ for planar scintigraphy.

Conclusion: Our results highlight the value of SPECT-CT in monitoring, quantitative evaluation of response to PRRT and its superiority in the prediction of decrease in tumour volume. The initial tumour uptake ratio from SPECT-CT could serve as a prognostic factor in the prediction of response to PRRT.
\end{abstract}

Keywords: 90Y-DOTATOC, 177Lu-DOTATOC, therapy monitoring, neuroendocrine tumour, SPECT/CT.

\section{INTRODUCTION}

A recent approach in the treatment of neuroendocrine tumours is the use of radiolabelled somatostatin analogues as Peptide Receptor Radionuclide Therapy (PRRT). The results obtained with ${ }^{90} \mathrm{Y}$ and ${ }^{177} \mathrm{Lu}$-DOTATOC are encouraging in terms of tumour regression and symptomatic improvement [1-7]. If reno-protective agents are used, the side effects of PRRT are few and transient.

Until now, monitoring and evaluating response to PRRT had been done by means of contrast enhanced CT and/or planar scintigraphy. The shortcomings of planar scintigraphy in providing precise anatomical localisation and problems due to overlay of lesions, often necessitate correlation with morphological imaging especially CT or MRI [8]. Correlation of morphological and functional imaging done

*Address correspondence to this author at the Department of Nuclear Medicine, University Hospital Basel, Petersgraben 4, CH-4031 Basel, Switzerland; Tel: +41-61-265 47 04; Fax: +41-61-265 48 97;

E-mail: guillaumen@uhbs.ch

${ }^{\S}$ These authors contributed equally to the work on a visual basis can lead to misinterpretation and errors, especially with respect to small abdominal lesions. Coregistration of anatomical and functional images by use of reference markers could improve retrospectively but not solve the alignment problems resulting from movement of internal organs entirely [9]. The only definitive solution to this problem is to acquire the anatomic and metabolic images simultaneously with the same imaging device and without moving the patient $[10,11]$. The benefit of fusion imaging using SPECT-CT in the diagnostic workup of neuroendocrine tumour has been proven and published in the recent years [10, 12-14].

To date there well defined criteria for monitoring and evaluating the response to PRRT [3, 15, 16]. Monitoring and evaluating the response to PRRT by SPECT-CT, combines the advantage of both morphological and functional quantification, and does not require nephrotoxic contrast agents. The primary aim of this retrospective study was to quantify the morphologic and functional response to PRRT by SPECT-CT. The secondary aim was to assess the value of SPECT-CT over planar scintigraphy in monitoring and predicting the outcome of PRRT. This is the first study to 
our knowledge, which monitors and evaluates the response of patients to PRRT using SPECT-CT Fusion Imaging.

\section{MATERIALS AND METHODS}

\section{Study Design}

A retrospective intra-personal comparison study was designed to evaluate the response of patients to PRRT by quantitative SPECT-CT analysis. The changes in the tumour uptake and volume after the first and second therapy on quantitatively evaluated. Planar scintigraphic images were analysed visually. ROC analysis was performed to assess the value of SPECT-CT over planar scintigraphy in the prediction of therapy outcome.

\section{Patient Population}

Inclusion of patients into the PRRT protocol was done by the following criteria: (a) histologically proven neuroendocrine tumour; (b) positive lesions on pretherapeutic octreoscan; (c) withdrawal of somatostatin analogues before therapy; (d) no previously described contraindications to therapy [2]. Between September 2005 and June 2006, patients were included into our study after applying the following inclusion criteria: underwent the first two courses of PRRT with ${ }^{90} \mathrm{Y}$ - or ${ }^{177} \mathrm{Lu}$-DOTATOC; underwent SPECT-CT imaging after the first and second therapy; and no other anti-neoplastic therapy in between the two courses of PRRT. All patients gave informed consent and the therapy protocol had been approved by the hospital ethics committee.

\section{Therapy Protocol}

The somatostatin analogues were synthesized in house according to a previously published procedure and radiolabelled with ${ }^{90} \mathrm{Y}$ or ${ }^{177} \mathrm{Lu}$ [17-19]. The infusion of Lysine $2.5 \%$ and Arginine $2.5 \%$ solution in 1 litre of $0.9 \%$ $\mathrm{NaCl}$ was started 30 minutes before the radiopeptide injection to reduce renal uptake. According to the protocol, patients were administered radiopeptide injection at a dose of $100 \mathrm{mCi} / \mathrm{m}^{2}\left(3700 \mathrm{MBq} / \mathrm{m}^{2}\right)$ (body surface area) of ${ }^{90} \mathrm{Y}$ DOTATOC for therapy and $3 \mathrm{mCi}(111 \mathrm{MBq})$ of ${ }^{111} \mathrm{In}$ DOTATOC for imaging purpose. ${ }^{177}$ Lu-DOTATOC was administered at a standard dose of $200 \mathrm{mCi}(7400 \mathrm{MBq})$. After 8-10 weeks, the second course of therapy was given, after controlling the haematological and renal parameters. When renal toxicity is documented, then a switch over to ${ }^{177} \mathrm{Lu}$-DOTATOC is made from ${ }^{90} \mathrm{Y}$-DOTATOC therapy.

\section{Follow-Up and Response}

All patients included into our study were evaluated at the end of 8-10 weeks after first therapy. SPECT-CT and planar images after the first and second therapy were retrospectively analysed. The decrease in tumour volume is considered as the favourable response to therapy.

\section{Imaging Protocol}

\section{Planar's Cintigraphy}

All planar images were acquired with a dual head gamma camera, Picker Prism 2000 XP. The windows were centered over both ${ }^{111}$ In photon peaks (172 and $\left.245 \mathrm{keV}\right)$ for
${ }^{90}$ Y-DOTATOC images and over ${ }^{177} \mathrm{Lu}$ peaks $(113$ and 208 $\mathrm{keV}$ ) for ${ }^{177} \mathrm{Lu}$-DOTATOC images with a width of $\pm 20 \%$. Parallel hole, medium energy, general purpose collimators were used. Imaging protocol intends to take planar images after 20-24 hours of radiopeptide injection. All planar scintigraphic images were evaluated by three board-certified experienced nuclear medicine physicians blinded with respect to the details of first or second therapy, date of acquisition, and patient data (Fig. 1). Initially, images of five reference cases not included into the study were distributed to the readers to assess the degree of inter-observer agreement and to familiarize them with the visual scoring system. The uptake of lesions in planar images was scored visually on a 5-point scale (Table 1). An overall score was also given for each patient.

\section{SPECT-CT}

24 hours after the radiopeptide injection, the SPECT-CT images were acquired focused on the areas of increased uptake in the previously acquired scintigraphic images. SPECT-CT imaging was performed using by Symbia T2 ${ }^{\circledR}$ (Siemens Inc.), a SPECT-CT system with an integrated dual detector variable angle gamma camera and a dual slice, noncontrast $\mathrm{CT}$ scanner. Non-contrast $\mathrm{CT}$ imaging provides the anatomical maps for attenuation correction of SPECT images. All images were reconstructed iteratively (OSEM3D, 4 subsets, 8 iterations), co-registered and evaluated using the e-soft ${ }^{\circledR}$ Syngo $^{\circledR}$ v5.1.4.6 (Siemens Inc.) software package. The SPECT images after first and second therapy were fused to localize the lesions and to make a visual comparison (Fig. 2). Then the SPECT, CT, and fusion images were evaluated after ensuring a precise coregistration by auto-registration and visual correction whenever necessary. The centre of the lesion was reached by correlating the uptake in SPECT with the CT lesion. SPECT, $\mathrm{CT}$ and fused axial slices were taken at same level as parallel range.

In the SPECT image, ROI was placed over the lesion and the mean and maximum uptake values were noted for each lesion (Fig. 3). This combines the advantage of little interference by statistical count rate fluctuations (owing to the use of mean ROI value) and interference by improper ROI placement in non-lesion areas (owing to the use of maximum ROI value). For each patient, the normal liver uptake (taken as background value) was measured by placing three ROIs in the tumour free liver as adjudged by the fusion images. Background ROIs were copied from fusion images to the SPECT images and to the second therapy images, to standardise the background variation. The mean liver background uptake was calculated by averaging the mean values from three background ROIs. Two ratios were calculated: LBR (Lesion Background ratio)-max, (maximum uptake value in the ROI/average liver background value), and LBR-mean (mean uptake value in the ROI/average liver background value). In case of patients with multiple liver metastases, three measurable and distinct lesions in both therapy images were taken as target lesions, and the ratios were calculated and averaged [20]. In case of non-liver lesions, the uptake was measured as above, and the background was taken from the normal liver irrespective of the location of the lesion. 


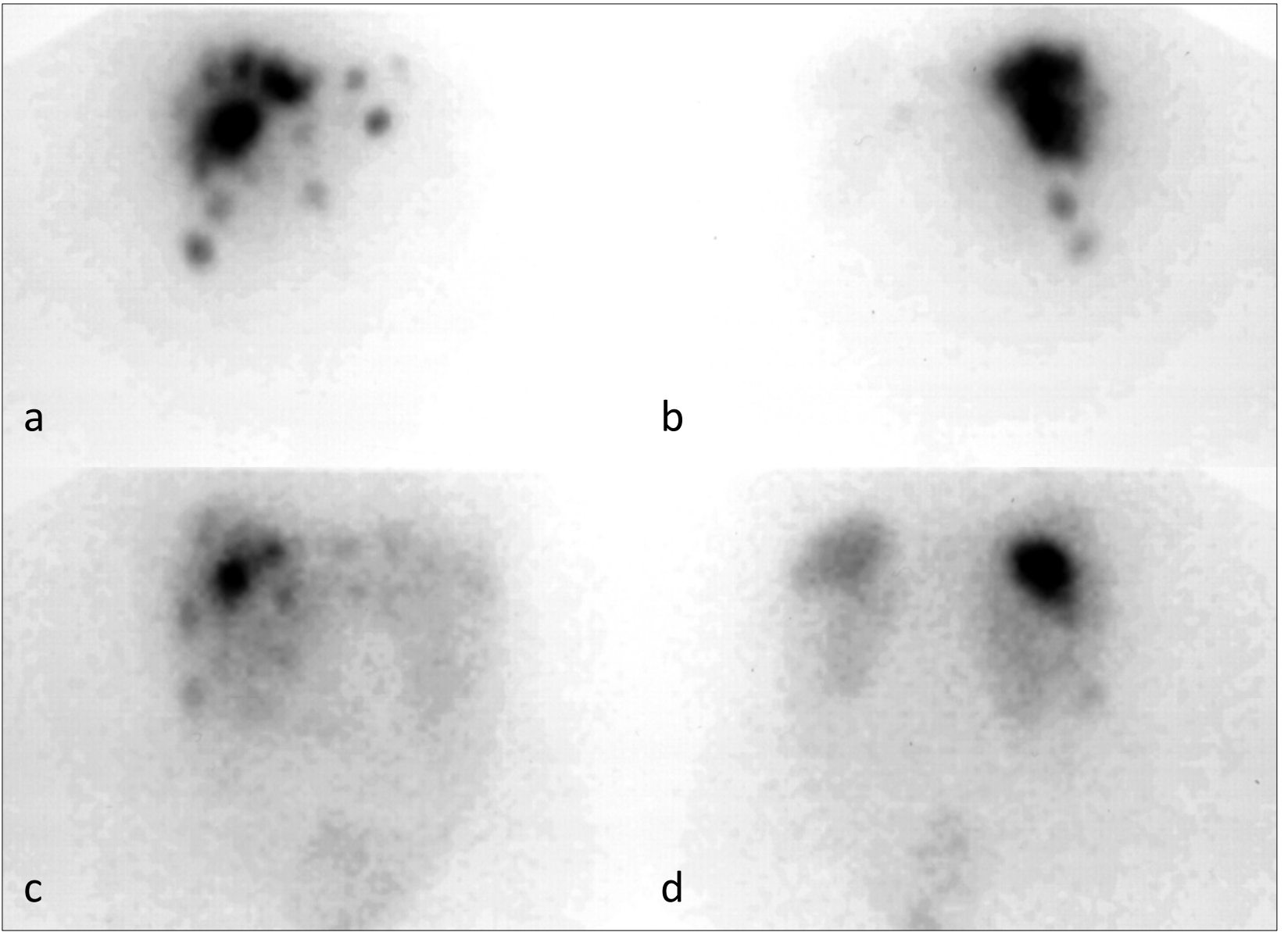

Fig. (1). Planar scintigraphic images after the first (a, b) ${ }^{90}$ Y-DOTATOC therapy and the second (c, d) ${ }^{90}$ Y-DOTATOC therapy. (a, c) Anterior and $(\mathbf{b}, \mathbf{d})$ posterior projections

In the axial CT image, the maximal diameter of the lesion and the largest perpendicular diameter were measured by using an electronic calliper tool in the same CT window settings $[20,21]$. In the coronal view, the maximal vertical dimension of the lesion was measured. The volume of the lesion was calculated by assuming the lesions to be ellipsoid and by the formula $[20,21]$ :

\section{$4 / 3 \times \pi \times($ maximal diameter/2) $\mathrm{x}$ (largest perpendicular diameter/2) $\mathrm{x}$ (maximal vertical diameter/2) $\mathrm{cm}^{3}$}

The change in the uptake ratios (biological response) and the lesion volume (morphological response) were quantified and compared with the first and the second therapy values.

\section{Grouping of Patients by Initial Tumour Uptake}

For objectivity and reproducibility of planar scintigraphy assessment, the following criteria were applied to the readers' scoring: patients who had scores of 1 or 2 were categorised into low uptake group. Those who had scores of 3 or 4 were categorised into as intermediate uptake, and those who had a score of 5 were categorised as the high uptake group. With reference to the initial tumour uptake ratio (LBR-max) from SPECT-CT, patients were stratified into three groups with respect to the $33.3^{\text {rd }}$ and $66.7^{\text {th }}$ percentile values. Rankings were given to the groups accordingly, Group 1 for low uptake, 2 for intermediate uptake, and 3 for high uptake group. We assessed and compared the trend of change in tumour uptake and volume in these three groups.

\section{Statistical Analysis}

All quantitative data were expressed as Mean \pm SD (In case of non-gaussian distribution, data were expressed as Median, Range). For paired comparison of non-parametric values, a Wilcoxon signed rank test was applied. The degree of agreement between the readers was measured with the Kendall's tau-b correlation coefficient as for ordinal-level variables. The value of quantitative SPECT-CT over visually assessed planar scintigraphy in the prediction of therapy response was evaluated by means of receiver operating characteristic (ROC) analysis [22]. Sensitivity analysis was done after excluding the patients treated with ${ }^{177} \mathrm{Lu}-$ DOTATOC and the results were compared with the values before exclusion. All statistical analyses were performed by using the statistical software package SPSS ${ }^{\circledR}$ v.14.0.0. All tests were of two-tailed significance with a $\mathrm{P}$ value of $<0.05$ indicating statistical significance. 
Table 1. Five Point Visual Assessment Score for Planar Scintigraphic Images

\begin{tabular}{|c|c|c|}
\hline Score & Lesion Uptake & Category \\
\hline \hline $\mathbf{1}$ & Lower than normal liver tissue uptake & Low uptake \\
\hline $\mathbf{2}$ & Equal to normal liver tissue uptake & Low uptake \\
\hline $\mathbf{3}$ & $\begin{array}{c}\text { Higher than normal liver tissue uptake and } \\
\text { lower than normal spleen/kidney uptake }\end{array}$ & $\begin{array}{c}\text { Intermediate } \\
\text { uptake }\end{array}$ \\
\hline $\mathbf{4}$ & Equal to normal spleen/kidney uptake & $\begin{array}{c}\text { Intermediate } \\
\text { uptake }\end{array}$ \\
\hline $\mathbf{5}$ & Higher than normal spleen/kidney uptake & High uptake \\
\hline
\end{tabular}

\section{RESULTS}

\section{Patient Population}

Between September 2005 and June 2006, 94 patients were referred to our department for radiolabelled DOTATOC therapy for neuroendocrine tumours. Of these cohort, 32 patients (23 males, 9 females, mean \pm SD age 60.3 \pm 12 years) were eligible to participate in described here study after applying the inclusion criteria. Fifteen patients had their primary lesion in pancreas, eight in ileum, two in lung, and one each in adrenal gland, duodenum, jejunum. Four patients had undiagnosed primary lesion.

\section{Radio-Peptide Therapy}

In the first course of therapy, 31 out of 32 patients, were injected with a median dose of $6,845 \mathrm{MBq}(186 \mathrm{mCi})$ of ${ }^{90} \mathrm{Y}-$ DOTATOC and $111 \mathrm{MBq}$ (3 mCi) of 111 In-DOTATOC (for imaging) and one patient was injected with 7,215 MBq (195 $\mathrm{mCi})$ of ${ }^{177} \mathrm{Lu}$-DOTATOC. As second therapy, 30 patients were injected with a median dose of $6,845 \mathrm{MBq}(186 \mathrm{mCi})$ of ${ }^{90} \mathrm{Y}$-DOTATOC and two patients were injected with $7,400 \mathrm{MBq}(200 \mathrm{mCi})$ of ${ }^{177} \mathrm{Lu}-$ DOTATOC. The average interval between two therapies was $61 \pm 8$ days.

\section{Therapy Response}

Seventy-seven target lesions were evaluated in 32 patients using SPECT. Out of these, only 67 lesions in 23 patients were measurable by $\mathrm{CT}$, and therefore included in the response evaluation by SPECT-CT. The calculated tumour volume from CT decreased from a median of 58.5 $\mathrm{cm}^{3}$ (range, 14.1 to 411.7 ) to $38.3 \mathrm{~cm}^{3}$ (9.9 to 428.4 ) with an overall decrease of $35 \%$. ( $\mathrm{P}=0.001$, Fig. $4 \mathbf{b})$. Thus, according to the volume criteria, two out of 23 patients showed partial response (9\%) and 21 patients showed stable disease $(91.31$ $\%)$.

\section{Planar Scintigraphy}

For inter-observer agreement, the Kendall's tau-b nonparametric correlation coefficients values were $0.794,0.847$, and 0.939 between the three readers $(\mathrm{P}<0.01)$. Visual assessment of planar scintigraphic images after the first therapy grouped 25 patients in the high uptake, five in the intermediate uptake, and two in the low uptake group. After the second therapy, this distribution changed to 20 patients in the high uptake, 10 in the intermediate, and two in the low uptake group.

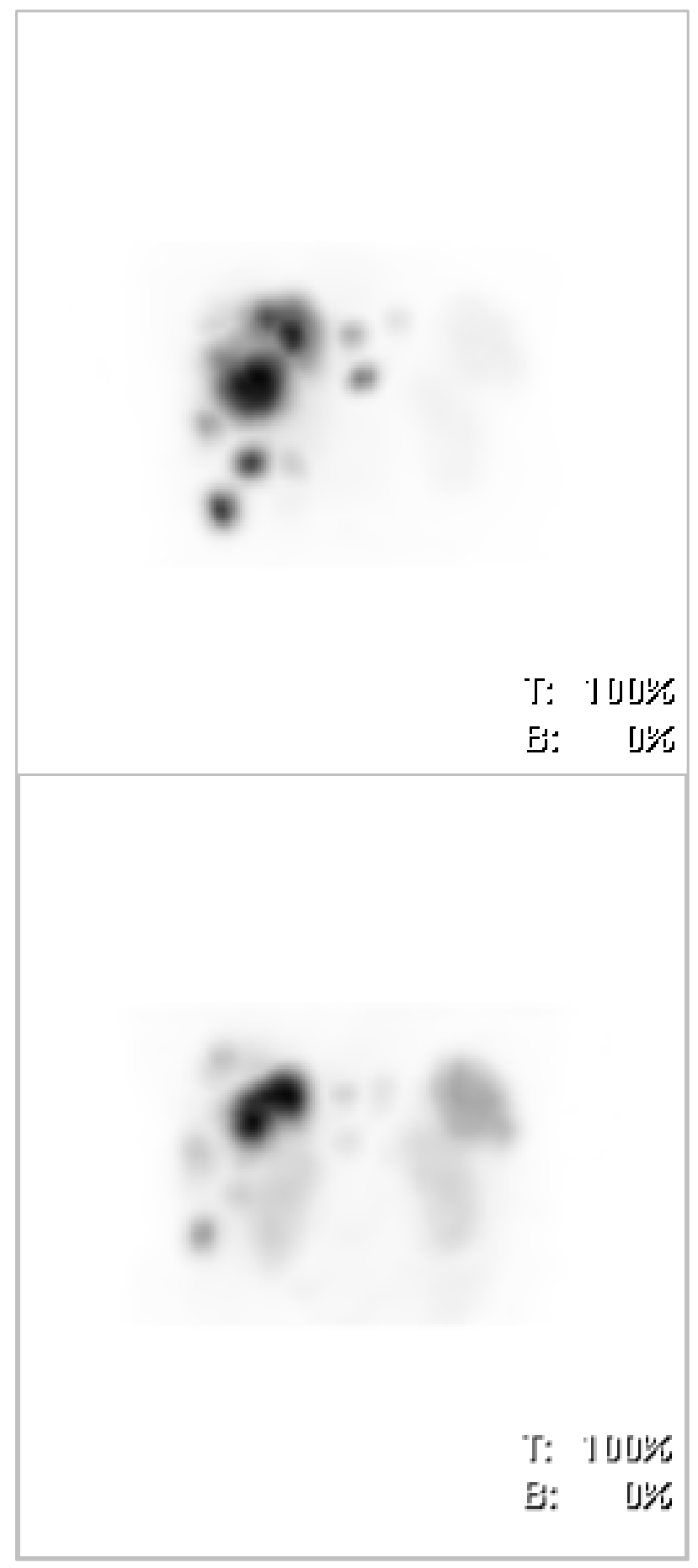

Fig. (2). SPECT Maximum Intensity Projection Image after the first therapy (above) and the second therapy (below).

\section{SPECT-CT}

Evaluation of 77 target lesions (in 32 patients) by SPECT-CT imaging showed that the mean uptake ratio (LBR-mean) decreased from a median value of 5.66 (range, 0.5 to 32.37$)$ at the first therapy images to 3.85 (0.95 to $15.75)$ at the second therapy, a $26 \%$ decrease $(\mathrm{P}<0.001)$. The maximum uptake ratio (LBR-max) decreased from the median of 7.98 (range, 0.97 to 54.21 ) to 5.59 (1.95 to 29.71), a $30 \%$ decrease $(\mathrm{P}<0.002$, Fig. 4a). 


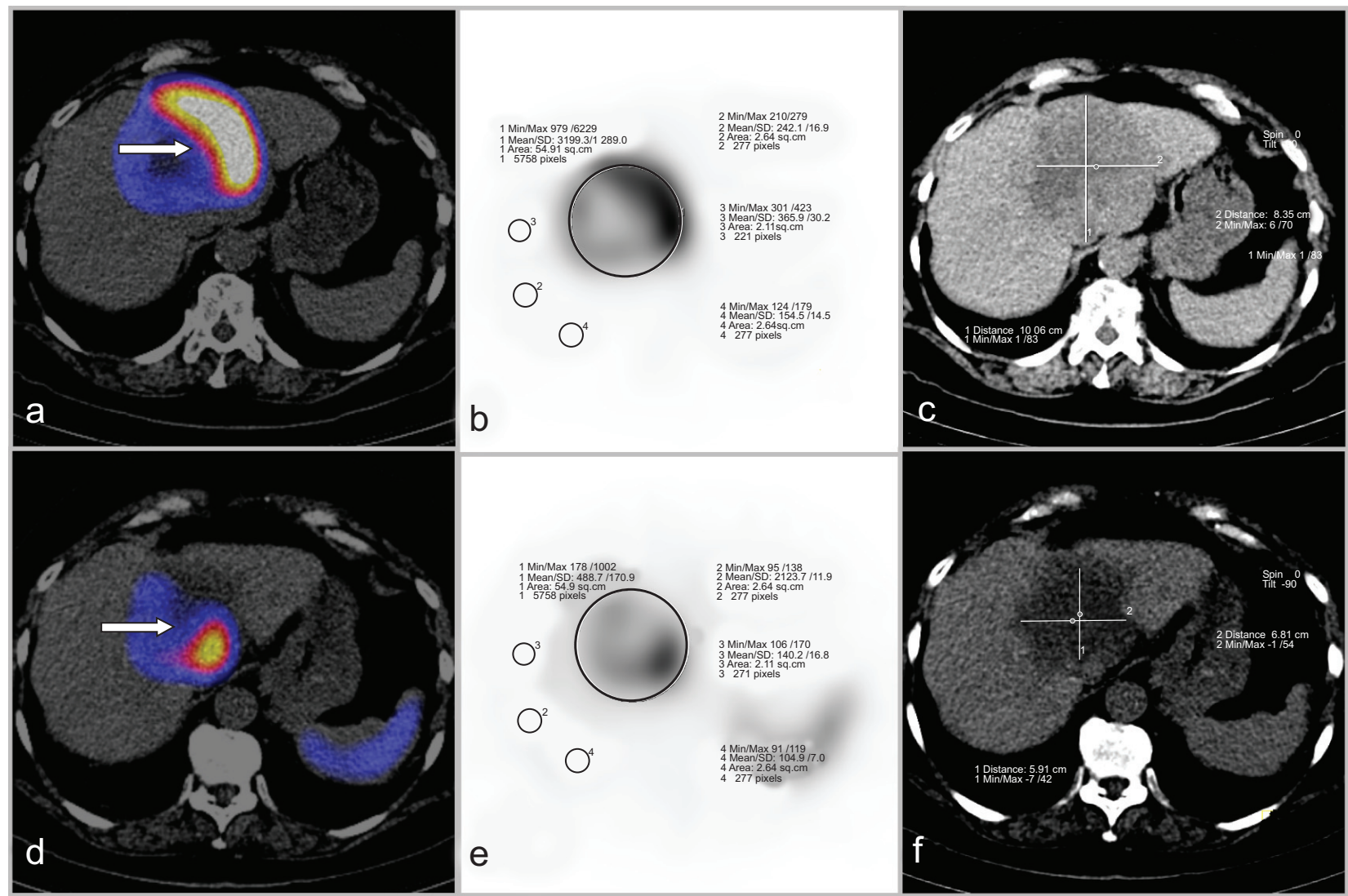

Fig. (3). Fusion (a), SPECT (b) and CT (c) images after the first course of ${ }^{90}$ Y-DOTATOC therapy. Note the change in uptake and dimensions in the same lesion (arrow) after the second course of ${ }^{90} \mathrm{Y}$-DOTATOC therapy (d-f).

\section{SPECT vs Planar Scintigraphy in the Prediction of Therapy Outcome}

The area under the ROC curve for SPECT-CT is found to be 0.733 (95\% Confidence Intervals: $0.203,0.819)$ compared to $0.551(95 \%$ Confidence Intervals: $0.485,0.981)$ for the planar imaging $(\mathrm{P}=0.16)$ (Fig. 5). The analysis of ROC curve showed that SPECT-CT is significant in predicting the response to PRRT $(\mathrm{P}=0.045)$. From the coordinates of the ROC curve, we could infer that a threshold value of 9.07 (LBR-max) corresponds to a sensitivity of $61 \%$ and specificity of $80 \%$ in predicting the decrease in tumour volume after PRRT. Our grouping values of LBR-max 6.81 $\left(33.3^{\text {rd }}\right.$ percentile) and $15.16\left(66.7^{\text {th }}\right.$ percentile) have a sensitivity and specificity of $80 \%, 60 \%$ and $40 \%, 80 \%$, respectively, in predicting a decrease in tumour volume. The ROC analysis of SPECT-CT and planar imaging curves after excluding three patients treated with ${ }^{177}$ Lu-DOTATOC revealed similar results with the $P$ values of 0.15 and 0.18 before and after exclusion.

\section{Results in Three Groups}

Considering initial uptake (LBR-max) values at first therapy, patients were categorized into three groups: 11 in the low uptake, 10 in the intermediate uptake, and 11 in the high uptake group. After the second therapy, this distribution changed to 18 patients in the low uptake, eight in the intermediate uptake, and six in the high uptake group. The quantitative SPECT-CT analysis of these three groups revealed in the group 3 (with high initial uptake) a median decrease in tumour uptake ratio (LBR-max) from 22.33 to 15.3 (decrease by 7.03 units) $(\mathrm{P}=0.006)$; in the group 2 a median decrease from 9.91 to 5.56 (decrease by 4.35 units) $(\mathrm{P}=0.005)$; and in the group 1 , a statistically insignificant decrease from 5.65 to 4.52 (decrease by 1.13 units) $(\mathrm{P}=$ 0.182 ) (Fig. 6). The tumour volume decreased in the group 3 by $67 \%$ from 92.9 to $31.1 \mathrm{~cm}^{3}(\mathrm{P}=0.036)$; in the group 2 by $37 \%$ from 61.6 to $39.0 \mathrm{~cm}^{3}(\mathrm{P}=0.063)$; and in the group 1 by $11 \%$ from 47.8 to $42.7 \mathrm{~cm}^{3}(\mathrm{P}=0.327)$.

\section{DISCUSSION}

The SPECT-CT based quantification of response to PRRT revealed a significant decrease in the tumour uptake and tumour volume at the end of second therapy. Moreover, the ROC analysis shows that the SPECT-CT is valuable in predicting the decrease in tumour volume.

Various parameters have been assessed in the evaluation of response to PRRT. For example, Bushnell et al. developed a clinical response scoring system, which includes the patient's body weight, health status score, Karnofsky performance score, and tumour related symptom score. This 

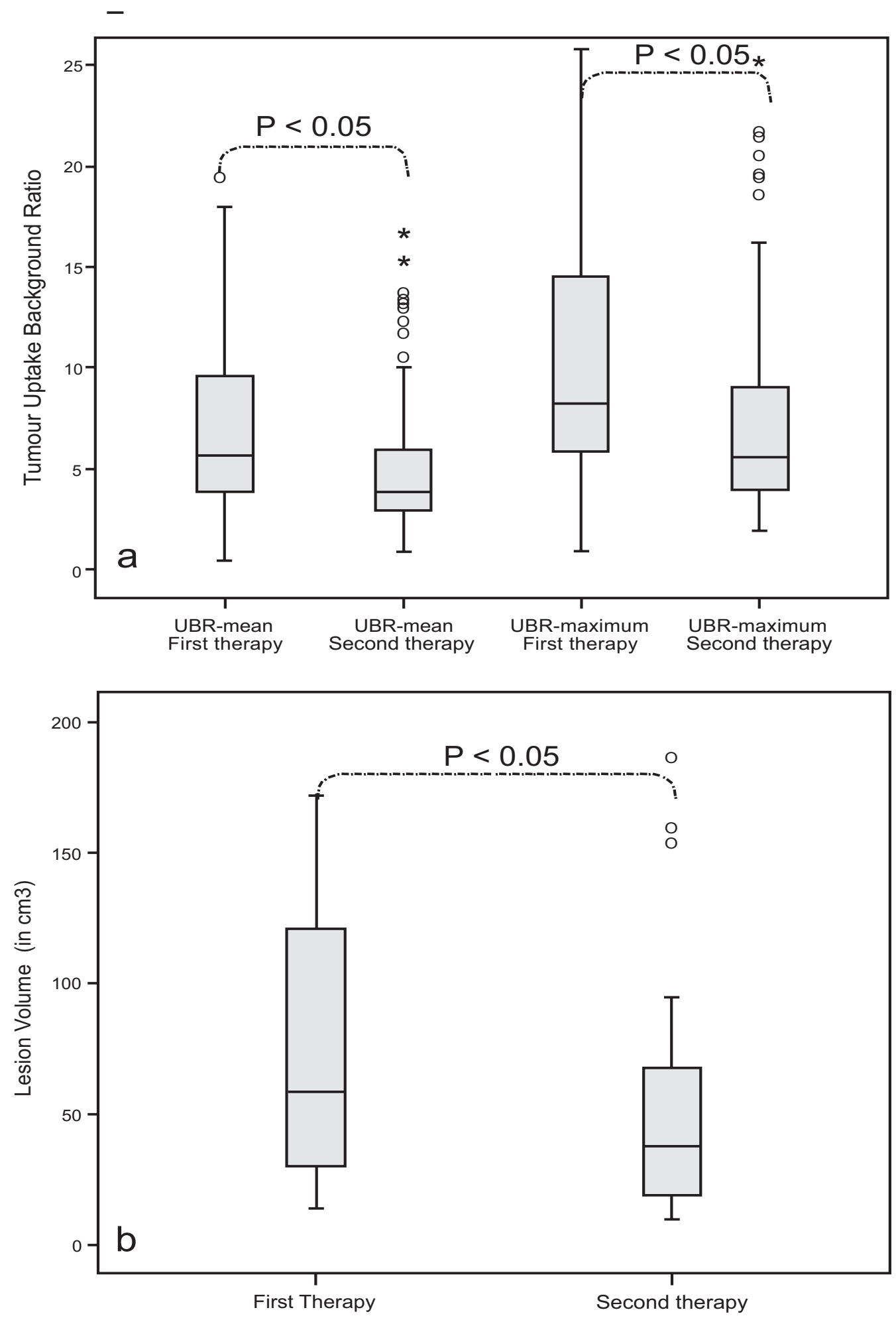

Fig. (4). Box and Whisker plots showing the decrease in tumour uptake as quantified in SPECT (a) and tumour dimensions as measured in CT (b) after the first and second therapy. (UBR - Uptake background ratio)

scoring system evaluated the impact of ${ }^{90} \mathrm{Y}-\mathrm{SMT} 487$ therapy on the clinical status of 21 patients [7]. This approach has the advantage to be easily accessible, inexpensive and allows a fair assessment of the clinical impact of such therapy. Unfortunately this scoring system lacks the necessary objectivity. However, CT examination (e.g. according to
RECIST criteria / WHO criteria) is limited too when attempting to determine the effectiveness of a cancer treatment. Other studies have shown the superiority of a functional restaging (as performed with FDG PET in lymphoma) over classical CT scan [23]. Kwekkeboom et al. evaluated response to ${ }^{177} \mathrm{Lu}$ - DOTATATE in 35 patients by 
correlating the effect of the tumour size with the tumour uptake in the planar scintigraphic images after scoring visually on a 4-point scale [3]. Nguyen et al. evaluated the efficacy of PRRT (using ${ }^{131} \mathrm{I}-\mathrm{MIBG}$ ) in 33 patients by assessing their clinical status, biochemical results, CT morphological changes and SRS, amount of dosage reduction in analgesic and cold somatostatin analogue [16]. Gopinath et al. showed in 22 patients that the functional tumour volume by SPECT quantification is more useful than $\mathrm{CT}$ in monitoring tumour response after therapy [24]. Most of the previous studies assessed the response based on planar, CT or SPECT images. In our study, the morphological and biological tumour responses to PRRT were quantified using SPECT-CT fusion imaging. The development of new somatostatine analogues with ${ }^{68} \mathrm{Ga}$ has paved the way for similar approach with PET/CT. Baum et al. using ${ }^{68} \mathrm{Ga}$-DOTANOC have measured tumor volume and quantified somatostatine receptor density in vivo before and after PRRT [25]. Although, the accuracy of PET/CT in measuring a standardised uptake value is attractive, dosimetry studies remain the gold standard in determining the delivered dose to lesions, but are not always practicable. In our case we took the advantage of using the routinely acquired data set from therapy patients, avoiding the need to perform any extra examination to the patients.

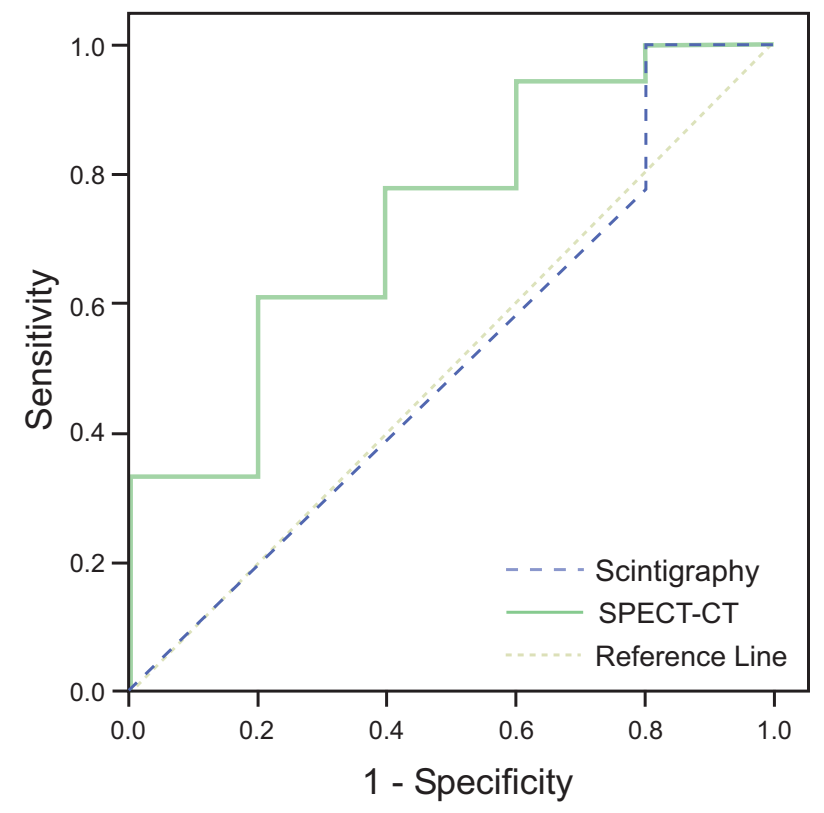

Fig. (5). ROC curves for the comparison of the prediction of outcome by SPECT-CT and planar scintigraphy.

This approach has several strengths but it also has its limitations: (i) the use of a dual slice, non-contrast, low dose $\mathrm{CT}$ in SPECT-CT may reduce the sensitivity in detecting the lesions and response. On the other hand, the contrast enhanced CT cannot provide precise attenuation correction for SPECT image due to dynamic contrast distribution. The non-contrast SPECT-CT allows precise correlation of SPECT lesions with corresponding lymph nodes or other anatomical structures in CT. (ii) Only a small group of patients treated with ${ }^{177} \mathrm{Lu}$-DOTATOC was evaluated in this study. For this reason, the results presented herein reflect mainly the response of patients to ${ }^{90} \mathrm{Y}$-DOTATOC therapy. (iii) The inclusion of patients treated with ${ }^{90} \mathrm{Y}$ - and ${ }^{177} \mathrm{Lu}$ DOTATOC may have introduced a selection bias in our study. However, the sensitivity analysis of ROC curves revealed similar results before and after exclusion of three patients treated with ${ }^{177}$ Lu-DOTATOC. (iv) In some nonliver lesions, we were unable to measure the normal lesion background. Therefore, the normal liver background was used instead, which might have altered the uptake ratio values for response evaluation. (v) Although the long-term follow-up data on the clinical impact of the therapy in these patients is lacking, the decrease in the uptake of the lesions correlates with the favourable clinical outcome [26-28].

Grouping of cases with Intial Uptake Background ratio value

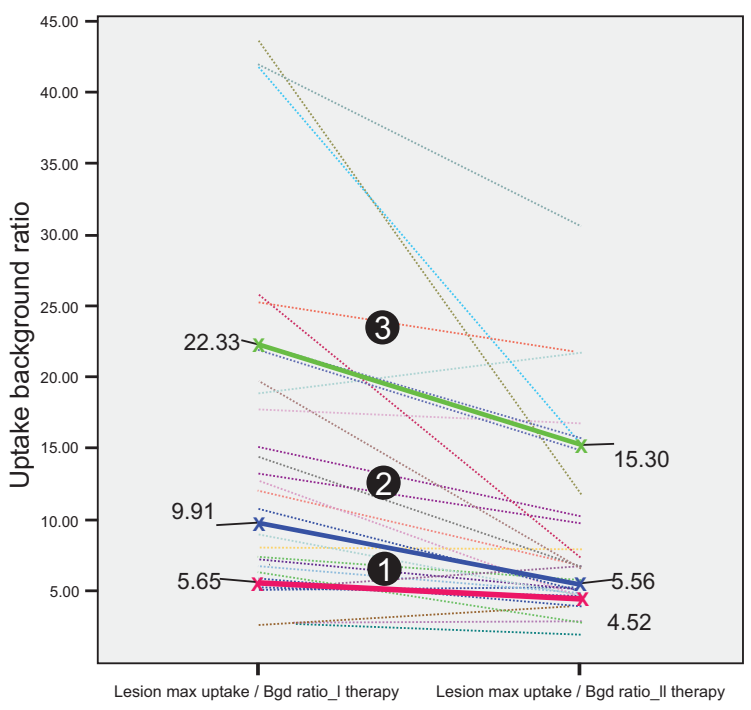

Fig. (6). Change in the uptake ratio (lesion background ratio -max) in lesions after the first and second therapy in three groups of patients stratified into Group 1 - Low uptake; Group 2 Intermediate uptake, and Group 3 - High uptake.

In this study, we performed a retrospective intra-personal comparison and quantified the morphological and biological response to PRRT by SPECT-CT fusion imaging. The exact anatomical localisation by $\mathrm{CT}$ with added functional receptor status of tumour enables an effective way to monitor the therapy. It appears that the lesion uptake background ratio obtained at the end of the first therapy may be a good prognostic factor of response to PRRT.

Our results show that there is a significant decrease in the tumour uptake and the tumour volume after PRRT. The higher the initial uptake in the lesion, the more significant decrease in the tumour uptake and volume is observed. This result is in concordance with the observation by Kwekkeboom et al. that the tumour response was significantly more frequent in patients with high uptake on the planar scintigraphy [3]. The SPECT-CT based quantification of response proves to be significant in the prediction of favourable decrease in the tumour volume. Even though the comparison of SPECT-CT and planar scintigraphy ROC curves did not show a statistically significant difference in the area under curves, the values for SPECT-CT are higher than for planar scintigraphy in the clinically relevant sensitivity and specificity ranges. 
Our study has several clinical implications: Quantitative SPECT-CT evaluation of patients would help in identifying the non-responders in the earliest and aids in the decision regarding modification of treatment protocol. Choosing the optimal threshold value for differentiating responders from non-responders could be defined by the point of the ROC curve with minimal distance from $100 \%$ True positive rate (sensitivity) and $0 \%$ False positive rate (1 - specificity) [29]. Ideally such decisions regarding threshold values should be made by linking the constructed ROC curve to explicit decision analysis and prospective randomised trials [22]. SPECT-CT paves a new way to monitor and quantify the response to PRRT in the clinical routine. The initial tumour uptake value would serve as a significant guide in stratifying patients and help in predicting prognosis and therapy outcome.

Further data are needed for evaluating and comparing the efficacy of the response to specific radio-pharmaceuticals using quantitative SPECT-CT. Further randomised prospective studies by quantitative SPECT-CT are necessary to obtain conclusions regarding the prognostic factors in predicting the outcome and real impact of PRRT in the management protocol of neuroendocrine tumours.

\section{CONCLUSION}

The results of our study highlight the quantitative evaluation of response to PRRT by SPECT-CT and its superiority in predicting the decrease in tumour volume. The initial tumour uptake ratio from SPECT-CT could serve as one of the prognostic factor in the prediction of response to PRRT.

\section{ACKNOWLEDGEMENTS}

The authors gratefully acknowledge and thank all the supporting personnel of the Department of Nuclear Medicine and Division of Radiological Chemistry, University Hospital Basel for their expert help and technical support.

\section{REFERENCES}

[1] Valkema R, Pauwels S, Kvols LK, et al. Survival and response after peptide receptor radionuclide therapy with [90YDOTA0,Tyr3]octreotide in patients with advanced gastroenteropancreatic neuroendocrine tumors. Semin Nucl Med 2006; 36: 147-56

[2] Kwekkeboom DJ, Mueller-Brand J, Paganelli G, et al. Overview of results of peptide receptor radionuclide therapy with 3 radiolabeled somatostatin analogs. J Nucl Med 2005; 46 Suppl 1: 62S-6S.

[3] Kwekkeboom DJ, Bakker WH, Kam BL, et al. Treatment of patients with gastro-entero-pancreatic (GEP) tumours with the novel radiolabelled somatostatin analogue [177 LuDOTA(0),Tyr3]octreotate. Eur J Nucl Med Mol Imaging 2003; 30: 417-22.

[4] Waldherr C, Pless M, Maecke HR, Haldemann A, Mueller-Brand J. The clinical value of [90Y-DOTA]-D-Phe1-Tyr3-octreotide (90YDOTATOC) in the treatment of neuroendocrine tumours: a clinical phase II study. Ann Oncol 2001; 12: 941-5.

[5] Iten F, Mueller B, Schindler C, et al. Response to [90YttriumDOTA]-TOC treatment is associated with long-term survival benefit in metastasized medullary thyroid cancer: a phase II clinical trial. Clin Cancer Res 2007; 13: 6696-702.

[6] Iten F, Mueller B, Schindler C, et al. [(90)Yttrium-DOTA]-TOC response is associated with survival benefit in iodine-refractory thyroid cancer: long-term results of a phase 2 clinical trial. Cancer 2009; 115: 2052-62.
[7] Bushnell D, O'Dorisio T, Menda Y, et al. Evaluating the clinical effectiveness of 90Y-SMT 487 in patients with neuroendocrine tumors. J Nucl Med 2003; 44: 1556-60.

[8] Pfannenberg AC, Eschmann SM, Horger M, et al. Benefit of anatomical-functional image fusion in the diagnostic work-up of neuroendocrine neoplasms. Eur J Nucl Med Mol Imaging 2003; 30: 835-43.

[9] Wahl RL, Quint LE, Cieslak RD, Aisen AM, Koeppe RA, Meyer CR. "Anatometabolic" tumor imaging: fusion of FDG PET with CT or MRI to localize foci of increased activity. J Nucl Med 1993; 34: 1190-7.

[10] Patton JA, Delbeke D, Sandler MP. Image fusion using an integrated, dual-head coincidence camera with X-ray tube-based attenuation maps. J Nucl Med 2000; 41: 1364-8.

[11] Shreve PD. Adding structure to function. J Nucl Med 2000; 41: 1380-2.

[12] Schillaci O, Scopinaro F, Danieli R, et al. Single photon emission computerized tomography increases the sensitivity of indium-111pentetreotide scintigraphy in detecting abdominal carcinoids. Anticancer Res 1997; 17: 1753-6.

[13] Schillaci O, Scopinaro F, Angeletti S, et al. SPECT improves accuracy of somatostatin receptor scintigraphy in abdominal carcinoid tumors. J Nucl Med 1996; 37: 1452-6.

[14] Even-Sapir E, Keidar Z, Sachs J, et al. The new technology of combined transmission and emission tomography in evaluation of endocrine neoplasms. J Nucl Med 2001; 42: 998-1004.

[15] Bushnell D, Menda Y, O'Dorisio T, et al. Effects of intravenous amino acid administration with Y-90 DOTA-Phe1-Tyr3-Octreotide (SMT487[OctreoTher) treatment. Cancer Biother Radiopharm 2004; 19: 35-41.

[16] Nguyen C, Faraggi M, Giraudet al, et al. Long-term efficacy of radionuclide therapy in patients with disseminated neuroendocrine tumors uncontrolled by conventional therapy. J Nucl Med 2004; 45: $1660-8$.

[17] Wild D, Schmitt JS, Ginj M, et al. DOTA-NOC, a high-affinity ligand of somatostatin receptor subtypes 2, 3 and 5 for labelling with various radiometals. Eur J Nucl Med Mol Imaging 2003; 30: 1338-47.

[18] Reubi JC, Schar JC, Waser B, et al. Affinity profiles for human somatostatin receptor subtypes SST1-SST5 of somatostatin radiotracers selected for scintigraphic and radiotherapeutic use. Eur J Nucl Med 2000; 27: 273-82.

[19] Reubi JC, Waser B, Schaer JC, Laissue JA. Somatostatin receptor sst1-sst5 expression in normal and neoplastic human tissues using receptor autoradiography with subtype-selective ligands. Eur J Nucl Med 2001; 28: 836-46.

[20] Therasse P, Arbuck SG, Eisenhauer EA, et al. New guidelines to evaluate the response to treatment in solid tumors. European Organization for Research and Treatment of Cancer, National Cancer Institute of the United States, National Cancer Institute of Canada. J Natl Cancer Inst 2000; 92: 205-16.

[21] James K, Eisenhauer E, Christian M, et al. Measuring response in solid tumors: unidimensional vs bidimensional measurement. J Natl Cancer Inst 1999; 91: 523-8.

[22] van Erkel AR, Pattynama PM. Receiver operating characteristic (ROC) analysis: basic principles and applications in radiology. Eur J Radiol 1998; 27: 88-94.

[23] Jerusalem G, Beguin Y, Fassotte MF, et al. Whole-body positron emission tomography using $18 \mathrm{~F}$-fluorodeoxyglucose for posttreatment evaluation in Hodgkin's disease and non-Hodgkin's lymphoma has higher diagnostic and prognostic value than classical computed tomography scan imaging. Blood 1999; 94: 429-33.

[24] Gopinath G, Ahmed A, Buscombe JR, Dickson JC, Caplin ME, Hilson AJ. Prediction of clinical outcome in treated neuroendocrine tumours of carcinoid type using functional volumes on $111 \mathrm{In}-$ pentetreotide SPECT imaging. Nucl Med Commun 2004; 25: 2537.

[25] Baum RP, Prasad V, Hommann M, Horsch D. Receptor PET/CT Imaging of Neuroendocrine Tumors. Recent Results Cancer Res 2008; 170: 225-42.

[26] De Jong M, Breeman WA, Bernard HF, et al. Therapy of neuroendocrine tumors with radiolabeled somatostatin-analogues. Q J Nucl Med 1999; 43: 356-66. 
[27] Virgolini I, Patri P, Novotny C, et al. Comparative somatostatin receptor scintigraphy using in-111-DOTA-lanreotide and in-111DOTA-Tyr3-octreotide $v s$ F-18-FDG-PET for evaluation of somatostatin receptor-mediated radionuclide therapy. Ann Oncol 2001; 12 Suppl 2: S41-5.
[28] Virgolini I, Traub T, Novotny C, et al. New trends in peptide receptor radioligands. Q J Nucl Med 2001; 45: 153-9.

[29] Stahl A, Ott K, Schwaiger M, Weber WA. Comparison of different SUV-based methods for monitoring cytotoxic therapy with FDG PET. Eur J Nucl Med Mol Imaging 2004; 31: 1471-8.

(c) Chinnadurai et al.; Licensee Bentham Open.

This is an open access article licensed under the terms of the Creative Commons Attribution Non-Commercial License (http: //creativecommons.org/licenses/by-nc/ 3.0/) which permits unrestricted, non-commercial use, distribution and reproduction in any medium, provided the work is properly cited. 